\title{
HOLOMORPHIC COHOMOLOGY OF COMPLEX ANALYTIC LINEAR GROUPS
}

\author{
G. HOCHSCHILD and G. D. MOSTOW \\ To the memory of Tadasi Nakayama
}

Introduction. Let $G$ be a complex analytic group, and let $A$ be the representation space of a finite-dimensional complex analytic representation of $G$. We consider the cohomology for $G$ in $A$, such as would be obtained in the usual way from the complex of holomorphic cochains for $G$ in $A$. Actually, we shall use a more conceptual categorical definition, which is equivalent to the explicit one by cochains. In the context of finite-dimensional representation theory, nothing substantial is lost by assuming that $G$ is a linear group. Under this assumption, it is the main purpose of this paper to relate the holomorphic cohomology of $G$ to Lie algebra cohomology, and to the rational cohomology, in the sense of [1], of algebraic hulls of $G$. This is accomplished by using the known structure theory for complex analytic linear groups in combination with certain easily established results concerning the cohomology of semidirect products. The main results are Theorem 4.1 (whose hypothesis is always satisfied by a complex analytic linear group) and Theorems 5.1 and 5.2. These last two theorems show that the usual abundantly used connections between complex analytic representations of complex analytic groups and rational representations of algebraic groups extend fully to the superstructure of cohomology.

1. Holomorphic maps. We recall some of the elementary facts concerning (Hausdorff) topological vector spaces over the field $C$ of the complex numbers. Every finite-dimensional subspace of such a topological vector space is closed and topologically isomorphic with a $C^{n}$. If $A$ is a locally convex topological vector space over $C$ then every neighborhood of 0 in $A$ contains a neighborhood of 0 that is not only convex but also stable under the multiplications with complex numbers of absolute value not exceeding 1 . Such a neighborhood is

Received May 31, 1965. 
called a disked convex neighborhood. We recall also that the continuous linear functionals on $A$ separate the points of $A$.

If the topological vector space $A$ is not only locally convex but also complete then there is available an adequate integration theory for $A$-valued functions (c.f. [0], Prop. 2, §4, and Remarque (1), pp. 81-82). Specifically, let $S$ be a compact topological space, and let $F(S, C)$ and $F(S, A)$ denote the spaces of all continuous maps of $S$ into $C$ or $A$, respectively. Suppose that $I: F(S, C)$ $\rightarrow C$ is an integral with the usual properties, i.e., $I$ is $C$-linear, commutes with the complex conjugation, sends non-negative real-valued functions onto non-negative real numbers and is continuous with respect to the uniform topology on $F(S, C)$. Then there is one and only one linear map $J: F(S, A)$ $\rightarrow A$ such that $\gamma(J(f))=I(\gamma \circ f)$ for every $f$ in $F(S, A)$ and every continuous linear functional $\gamma$ on $A$, and $J$ is continuous with respect to the uniform topology on $F(S, A)$.

We give a brief discussion of convergence of series in a complete locally convex topological vector space $A$. Let $K$ be a set of indices, and let $k \rightarrow a_{k}$ be a map of $K$ into $A$. We say that $\left(a_{k}\right)_{k \in K}$ is a Cauchy series in $A$ if, given any neighborhood $V$ of 0 in $A$, there is a finite subset $K(V)$ of $K$ such that, for every finite subset $L$ of $K$ not meeting $K(V), \sum_{k \in L} a_{k} \in V$. Since $A$ is complete, a Cauchy series $\left(a_{k}\right)_{k \in K}$ has a unique sum $\sum_{k \in K} a_{k}$ in $A$, which is determined by the property that, for every neighborhood $V$ of 0 in $A$, there is a finite subset $K^{*}(V)$ of $K$ such that $\sum_{k \in L} a_{k} \in V+\sum_{k \in K} a_{k}$ for every finite subset $L$ of $K$ that contains $K^{*}(V)$.

Now let us consider a formal integral power series $P\left(X_{1}, \ldots, X_{n}\right)=$ $\sum X_{1}^{e_{1}} \cdots X_{n}^{e_{n}} a\left(e_{1}, \ldots, e_{n}\right)$ with coefficients $a\left(e_{1}, \ldots, e_{n}\right)$ in $A$. Suppose that $b_{1}, \ldots, b_{n}$ are positive real numbers and that the series of terms $b_{1}^{e_{1}} \cdots b_{n}^{e_{n}}$ $a\left(e_{1}, \ldots, e_{n}\right)$ is a Cauchy series in $A$. Using that every neighborhood of 0 in $A$ contains a disked convex neighborhood of 0 , one sees easily that if $\left(c_{1}, \ldots, c_{n}\right)$ is any $n$-tuple of complex numbers such that $\left|c_{i}\right|<b_{i}$ for each $i$ then the series of terms $c_{1}^{e_{1}} \cdots c_{n}^{e_{n}} a\left(e_{1}, \ldots, e_{n}\right)$ is a Cauchy series, and thus has a unique sum $p\left(c_{1}, \ldots, c_{n}\right)$ in $A$. Thus $P$ determines a map $p$ of the open box $B$ in $C^{n}$ defined by the system of inequalities $\left|c_{i}\right|<b_{i}, i=1, \ldots, n$, into $A$. Moreover, one sees at the same time that the convergence of the family of finite sums of terms $c_{1}^{e_{1}} \cdots c_{n}^{e_{n}} a\left(e_{1}, \ldots, e_{n}\right)$ to $p\left(c_{1}, \ldots, c_{n}\right)$ is 
uniform on every compact subset of $B$. We shall call $p$ the power series map of $B$ into $A$ that is represented by the formal power series $P\left(X_{1}, \ldots, X_{n}\right)$. It is easily seen that the formal partial derivatives $D_{i}(P)\left(X_{1}, \ldots, X_{n}\right)$ and the formal partial antiderivatives $D_{i}^{-1}(P)\left(X_{1}, \ldots, X_{n}\right)$ again represent power series maps of $B$ into $A$, that the power series maps of $B$ into $C$ constitute a $C$-algebra $\mathbf{P}(B, C)$, and that the totality $\mathbf{P}(B, A)$ of all power series maps of $B$ into $A$ becomes a $\mathbf{P}(B, C)$-module in the natural way.

Now let $M$ be a complex analytic manifold. A map $f$ of $M$ into the complete locally convex topological vector space $A$ is called holomorphic if, for every point $m$ of $M$, there is a chart $\mu: V \rightarrow \mu(V) \subset C^{n}$ of $M$ at $m$ such that $f \circ \mu^{-1}$ is a power series map of $\mu(V)$ into $A$. If $S$ is an arbitrary topological vector space then by a holomorphic map of $M$ into $S$ we shall mean a finite sum of holomorphic maps of $M$ into complete locally convex subspaces of $S$. The space $F_{h}(M, S)$ of all holomorphic maps of $M$ into $S$ is a module over the $C$ algebra $F_{h}(M, C)$ of all holomorphic complex-valued functions on $M$ in the natural way.

If $\rho: S \rightarrow A$ is a continuous linear map of $S$ into a complete locally convex topological vector space $A$ then we have evidently $\rho \circ F_{h}(M, S) \subset F_{h}(M, A)$. Similarly, if $\eta: M \rightarrow N$ is an analytic map of $M$ into another complex analytic manifold $N$ then $F_{h}(N, S) \circ \eta \subset F_{h}(M, S)$. The Cauchy integral formulae are valid and can be used as usual to obtain the following facts. If $\mu: V \rightarrow B$ is a chart of $M$, with $B=\mu(V)$ an open box in $C^{n}$, and if $f$ is a holomorphic map of the open submanifold $V$ of $M$ into $S$ then $f \circ \mu^{-1}$ is actually a power series map of $B$ into $S$. If $A$ is a complete locally convex topological vector space then the compact-open topology makes $F_{h}(M, A)$ a complete locally convex topological vector space.

2. Holomorphic modules for complex analytic groups. Let $G$ be a complex analytic group. By a holomorphic G-module we mean a topological vector space $A$ over $C$, together with a continuous map $G \times A \rightarrow A$, written $(g, a) \rightarrow g \cdot a$, making $A$ into a continuous $G$-module in the usual sense and having the following additional property: if $a \in A$ and $f_{a}$ denotes the map of $G$ into $A$ given by $f_{a}(g)=g \cdot a$ then $f_{a}$ is a holomorphic map of $G$ into $A$. One sees easily that if $F_{h}(G, A)$ is endowed with the compact-open topology then the map $a \rightarrow f_{a}$ of $A$ into $F_{h}(G, A)$ is continuous. We make $F_{h}(G, A)$ into a continuous 
$G$-module by defining, for $f$ in $F_{h}(G, A)$ and $x$ in $G$, the transform $x \cdot f$ by $(x \cdot f)(y)=f(y x)$. It is not difficult to show that, actually, this makes $F_{h}(G, A)$ into a holomorphic $G$-module.

A holomorphic $G$-module $I$ is said to be holomorphically injective if it has the following property: let $V$ be a holomorphic $G$-module, let $U$ be a $G$-submodule of $V$ that is a direct topological vector space summand of $V$, and let $\mu$ be a continuous $G$-module homomorphism of $U$ into $I$; then $\mu$ can be extended to a continuous $G$-module homomorphism of $V$ into $I$. The following proposition is basic for our purposes.

Proposition 2.1. Let $G$ be a complex analytic group, and let $S$ be a complex analytic subgroup of $G$. Suppose that there is a complex analytic map $\rho: G \rightarrow S$ such that $\rho(g s)=\rho(g) s$ for every $g$ in $G$ and every $s$ in $S$. Let $A$ be a complete locally convex topological vector space over $C$. Then the holomorphic S-module $F_{h}(G, A)$ is holomorphically injective.

Proof. Let $V$ be a holomorphic $S$-module, and let $\sigma$ be a continuous linear projection of $V$ onto an $S$-submodule $U$ of $V$. Let $\mu$ be a continuous $S$-module homomorphism of $U$ into $F_{h}(G, A)$. We must show that $\mu$ can be extended to a continuous $S$-module homomorphism of $V$ into $F_{h}(G, A)$. For every element $v$ of $V$, define the map $\gamma(v)$ of $G$ into $A$ by $\gamma(v)(x)=\mu(\sigma(\rho(x) \cdot v))\left(x \rho(x)^{-1}\right)$. The map $x \rightarrow \rho(x) \cdot v$ is a holomorphic map of $G$ into $V$. Since $\mu^{\circ} \sigma$ is continuous and $F_{h}(G, A)$ is locally convex and complete, it follows that the map $x \rightarrow \mu(\sigma(\rho(x) \cdot v))$ is a holomorphic map of $G$ into $F_{h}(G, A)$, whence we see that $\gamma(v) \in F_{h}(G, A)$. Moreover, $\gamma$ is evidently a linear map, and it is easy to see that it is continuous. Finally, one verifies directly that $r$ coincides with $\mu$ on $U$ and that $\gamma$ is an $S$-module homomorphism, which completes the proof of Proposition 2.1.

Lemma 2.2. Let $Q$ be a closed connected subgroup of the complex analytic group $G$, and let $Q^{*}$ denote the smallest closed complex analytic subgroup of $G$ containing $Q$. Suppose that $f$ is a holomorphic map of $G$ into the complete locally convex topological vector space $A$ such that $f(Q)=(0)$. Then also $f\left(Q^{*}\right)=(0)$.

Proof. It suffices to show that $(\gamma \circ f)\left(Q^{*}\right)=(0)$ for every continuous complex linear functional $r$ on $A$. Hence we may assume without loss of generality that $f$ is an ordinary complex-valued holomorphic function on $G$. Let $L$ denote 
the complex analytic subgroup of $G$ whose Lie algebra is $C \mathscr{L}(Q)$, where $\mathscr{L}(Q)$ is the real Lie subalgebra of the Lie algebra of $G$ that corresponds to the real analytic subgroup $Q$ of $G$. Since $f(Q)=(0), f$ is annihilated by the canonical action of $\mathscr{L}(Q)$. Hence $f$ is annihilated also by the Lie algebra $C \mathscr{L}(Q)$ of $L$, whence $f$ is constant on $L$, so that $f(L)=(0)$. Hence $f$ vanishes also on the closure of $L$ in $G$. Repeating this argument with the closure of $L$ in $G$ taking the place of $Q$, etc., we reach the conclusion $f\left(Q^{*}\right)=(0)$ in a finite number of such steps. Thus Lemma 2.2 is established.

In the notation of Lemma 2.2, we shall say that $Q$ is an ample subgroup of $G$ if $Q^{*}=G$. Our results on the holomorphic cohomology of complex analytic groups are based on the following proposition.

Proposition 2.3. Suppose that the complex analytic group $G$ has an ample connected compact subgroup $Q$. Then every complete locally convex holomorphic $G$ module is holomorphically injective.

Proof. Let $A$ be a complete locally convex holomorphic $G$-module, and let $V$ be a holomorphic $G$-module having a continuous linear projection $\sigma$ onto a $G$-submodule $U$. Let $\mu$ be a continuous $G$-module homomorphism of $U$ into $A$. We must show that $\mu$ extends to a continuous $G$-module homomorphism of $V$ into $A$.

For every element $v$ of $V$, define the map $\eta_{v}$ of $Q$ into $A$ by $\eta_{v}(q)=$ $q^{-1} \cdot \mu(\sigma(q \cdot v))$. Clearly, $\eta_{v}$ lies in the space $F(Q, A)$ of all continuous maps of $Q$ into $A$, and the map $v \rightarrow \eta_{v}$ is continuous with respect to the uniform topology on $F(Q, A)$. Note that $F(Q, A)$ is complete and locally convex, and let $J_{Q}$ denote the $A$-valued integral on $F(Q, A)$ that corresponds to the normalized Haar integral on $F(Q, C)$. Then the map $\rho$ defined by $\rho(v)=J_{Q}\left(\eta_{v}\right)$ is a continuous linear map of $V$ into $A$. If $v$ lies in $U$ then $\eta_{v}$ is the constant map $Q \rightarrow A$ with value $\mu(v)$, whence $\rho$ coincides with $\mu$ on $U$. It remains only to show that $\rho$ is a $G$-module homomorphism.

If $q^{*}$ denotes the linear automorphism of $A$ effected by the element $q$ of $Q$ we have $\eta_{a \cdot v}=q^{*} c\left(q \cdot \eta_{v}\right)$. Hence $J_{Q}\left(\eta_{q \cdot v}\right)=q \cdot J_{Q}\left(q \cdot \eta_{v}\right)$. Because of the translation invariance of the Haar integral, we have $J_{Q}\left(q \cdot \eta_{v}\right)=J_{Q}\left(\eta_{v}\right)$. Hence $\rho(q \cdot v)$ $=q \cdot \rho(v)$. Now consider the map $\tau_{v}: G \rightarrow A$ defined by $\tau_{v}(x)=x \cdot \rho(v)-\rho(x \cdot v)$. Clearly, $\tau_{v}$ is holomorphic. Our last result means that $\tau_{v}(Q)=(0)$. By Lemma 
2.2, we have therefore $\tau_{v}=0$, which means that $\rho$ is a $G$-module homomorphism. This completes the proof of Proposition 2.3.

An exact sequence $\cdots \rightarrow A_{i} \rightarrow A_{i} A_{i+1} \rightarrow \cdots$ of holomorphic $G$-modules $A_{i}$ and continuous $G$-module homomorphisms $\alpha_{i}$ is said to be strongly exact if the kernel of each $\alpha_{i}$ is a direct topological vector space summand of $A_{i}$. Let $A$ be a complete locally convex holomorphic $G$-module, and let $\alpha$ be the map $A \rightarrow F_{h}(G, A)$ given by $\alpha(a)=f_{a}$, where $f_{a}(x)=x \cdot a$ for every $x$ in $G$. Evidently, $\alpha$ is a continuous $G$-module monomorphism. Moreover, the map $f \rightarrow f(1)$ is a continuous linear map of $F_{h}(G, A)$ into $A$ sending each $f_{a}$ back onto $a$. By Proposition $21, F_{h}(G, A)$ is a holomorphically injective holomorphic $G$-module. Moreover, $F_{h}(G, A)$ is complete and locally convex, whence $F_{h}(G, A) / \alpha(A)$, which is isomorphic to a direct topological vector space summand of $F_{h}(G, A)$, is also complete and locally convex. Clearly, it follows that $F_{h}(G, A) / \alpha(A)$ is a holomorphic $G$-module. By iterating the above construction, with $F_{h}(G, A) / \alpha(A)$ in the place of $A$, etc., we obtain a strongly exact sequence $(0) \rightarrow A \rightarrow X_{0}(A) \rightarrow X_{1}(A) \rightarrow \cdots$, where each $X_{i}(A)$ is a holomorphically injective holomorphic $G$-module. We call such a sequence a holomorphically injective resolution of $A$. From such a resolution, we obtain a complex of topological vector spaces $(0) \rightarrow X_{0}(A)^{G} \rightarrow X_{1}(A)^{G} \rightarrow \cdots$, where $X_{i}(A)^{G}$ denotes the $G$-fixed part of $X_{i}(A)$. The homology space $H\left(X(A)^{G}\right)$ of this complex is, to within natural isomorphisms, independent of the particular choice of the resolution $X(A)$. We denote it by $H_{h}(G, A)$ and call it the holomorphic cohomology space for $G$ in $A$.

3. Semidirect products. We prove a theorem which reduces the determination of the holomorphic cohomology of a linear complex analytic group to the case of a simply connected solvable complex analytic group.

Theorem 3.1. Suppose that the complex analytic group $G$ is a semidirect product $P \cdot K$, where $K$ is normal in $G$ and $P$ has a connected compact ample subgroup $Q$. Let $A$ be a complete locally convex holomorphic G-module. Then, for each dimension $n$, the cohomology restriction map of $H_{h}^{n}(G, A)$ into the G-fixed part $\left(H_{h}^{n}(K, A)\right)^{G}$ of the holomorphic cohomology space for $K$ in $A$ is a topological vector space isomorphism of $H_{h}^{n}(G, A)$ onto a subspace of $\left(H_{h}^{n}(K, A)\right)^{G}$. If $H_{h}^{n}(K, A)$ is a finite-dimensional Hausdorff space then the restriction image of $H_{h}^{n}(G, A)$ coincides with $\left(H_{h}^{n}(K, A)\right)^{\text {a }}$. 
Proof. Let $(0) \rightarrow A, X_{0} \rightarrow X_{1} \rightarrow \cdots$ be a holomorphically injective resolution of the holomorphic $G$-module $A$ such that each $X_{i}$ is complete and locally convex; we have seen at the end of Section 2 that such a resolution exists. Since $X_{i}$ is holomorphically injective, it is a direct $G$-module and topological vector space summand of $F_{h}\left(G, X_{i}\right)$. Since $X_{i}$ is complete and locally convex, so is $F_{h}\left(G, X_{i}\right)$. Hence we may apply Proposition 2.1 to conclude that $F_{h}\left(G, X_{i}\right)$ is holomorphically injective also as a $K$-module. The same is therefore true for $X_{i}$, so that our above resolution is also a holomorphically injective resolution of $A$ as a holomorphic $K$-module. Hence the restriction map $H_{h}(G, A)$ $\rightarrow\left(H_{h}(K, A)\right)^{G}$ is realized by the map $\rho: H\left(X^{G}\right) \rightarrow H\left(X^{K}\right)$ that is induced by the injection $X^{G} \rightarrow X^{K}$. For an element $x$ of an $X_{i}^{K}$, denote by $f_{x}$ the map of $Q$ into $X_{i}^{K}$ given by $f_{x}(q)=q \cdot x$. Let $J$ denote the integral on $F\left(Q, X_{i}^{K}\right)$ that corresponds to the normalized Haar integral on $Q$ (note that $X_{i}^{K}$ is complete and locally convex, because it is closed in $\left.X_{i}\right)$. Now put $\tau(x)=J\left(f_{x}\right)$. Then $\tau(x)$ is a $Q$-fixed element of $X_{i}^{K}$, and it follows from Lemma 2.2 that $\tau(x)$ is actually $P$-fixed. Hence $\tau(x) \in X_{i}^{(\xi}$ and it is now clear that $\tau$ is a continuous linear projection of $X_{i}^{K}$ onto $X_{i}^{G}$. Moreover, if $d_{i}$ is the given $G$-module homomorphism $X_{i} \rightarrow X_{i+1}$ we have $d_{i} \circ f_{x}=f_{d_{i}(x)}$, whence $d_{i} \circ \tau=\tau \circ d_{i}$. Hence $\tau$ induces a continuous linear map $\tau^{*}: H\left(X^{\kappa}\right) \rightarrow H\left(X^{\sigma}\right)$, and evidently $\tau^{*} \circ \rho$ is the identity map on $H\left(X^{\alpha}\right)$. This establishes the first part of Theorem 3.1.

Now let $Z\left(X_{n}^{K}\right)$ denote the kernel of $d_{n}$ in $X_{n}^{K}$, and consider the exact sequence $(0) \rightarrow d_{n-1}\left(X_{n-1}^{K}\right) \rightarrow Z\left(X_{n}^{K}\right) \rightarrow H_{h}^{n}(K, A) \rightarrow(0)$. If $H_{h}^{n}(K, A)$ is a finitedimensional Hausdorff space the exactness here implies that $d_{n-1}\left(X_{n-1}^{K}\right)$ is a direct topological vector space summand of $Z\left(X_{n}^{K}\right)$. Since $Z\left(X_{n}^{K}\right)$ is a closed $P$-submodule of $X_{n}$, it is a holomorphic $P$-module. Hence $d_{n-1}\left(X_{n-1}^{K}\right)$ is also a holomorphic $P$-module. Moreover, it is complete and locally convex. By Proposition 2.3, it is therefore holomorphically injective as a $P$-module. Hence it is not only a direct topological vector space summand of $Z\left(X_{1 b}^{K}\right)$ but also a direct holomorphic $P$-module summand. Hence it is evident that $\left(H_{h}^{n}(K, A)\right)^{P}$ is the image of $Z\left(X_{n}^{K}\right)^{P}$, i.e., that $\left(H_{h}^{n}(K, A)\right)^{G}$ is the image of $Z\left(X_{n}^{(A)}\right)$, so that the restriction $\operatorname{map} H_{h}^{\eta}(G, A) \rightarrow\left(H_{h}^{\eta}(K, A)\right)^{G}$ is surjective. This completes the proof of Theorem 3.1.

4. Simply connected solvable analytic groups. We must briefly review the basic facts concerning the holomorphic differential forms on a complex analytic 
group $G$. The details we omit can be found in $[3, \S 6]$. Let $T$ denote the $F_{h}(G, C)$-module consisting of the holomorphic tangent vector fields on $G$. The group $G$ acts on $T$ from the right as follows. Let $\tau$ be an element of $T$, and let $x$ be an element of $G$. Then the transform $\tau \cdot x$ is defined by associating with each open subset $U$ of $G$ the corresponding derivation $(\tau \cdot x)_{U}$ of the $C$ algebra of the holomorphic functions on $U$. If $f$ is such a function we put $(\tau \circ x)_{U}(f)=\tau_{x^{-1} U}\left(f \circ t_{x}\right) \circ t_{x}^{-1}$, where $t_{x}$ denotes the translation $y \rightarrow x y$ that is effected by $x$ on $G$. Then the Lie algebra $\mathscr{L}(G)$ of $G$ consists precisely of the $G$-fixed elements of $T$, and the canonical map of the tensor product $F_{h}(G, C) \otimes r \mathscr{L}(G)$ into $T$ is bijective.

Let $A$ be a complete locally convex holomorphic $G$-module. For every positive integer $q$, Jet $D^{q}(T, A)$ denote the $F_{h}(G, C)$-module of all $F_{h}(G, C)$ multilinear alternating maps of the $q$-tuples of elements of $T$ into $F_{h}(G, A)$. We put $D^{o}(T, A)=F_{h}(G, A)$, and we let $D(T, A)$ denote the direct sum of the $F_{h}(G, C)$-modules $D^{a}(T, A)$. The elements of $D(T, A)$ are called the holomorphic differential forms for $G$ in $A$. Now $D(T, A)$ is made into a complex by introducing the differential operator $\delta: D^{q}(T, A) \rightarrow D^{q+1}(T, A)$ given by the familiar formula

$$
\begin{aligned}
& \delta(\alpha)\left(\tau_{0}, \ldots, \tau_{q}\right)=\sum_{i=0}^{q}(-1)^{i} \tau_{i}\left(\alpha\left(\tau_{0}, \ldots, \hat{\tau}_{i}, \ldots, \tau_{q}\right)\right. \\
& \quad+\sum_{r<s}(-1)^{r+s} \alpha\left(\left[\tau_{r}, \tau_{s}\right], \tau_{0}, \ldots, \hat{\tau}_{r}, \ldots, \hat{\tau}_{s}, \ldots, \tau_{q}\right)
\end{aligned}
$$

Here, $\tau(f)$, with $\tau$ in $T$ and $f$ in $F_{h}(G, A)$, is the element of $F_{h}(G, A)$ that is obtained by applying $\tau$ locally to the power series functions obtained from the restrictions of $f$ to domains of suitable charts of $G$.

We define a right $G$-module structure on $F_{h}(G, A)$ by $(f \cdot x)(y)=x^{-1} \cdot f(x y)$. Then one defines a $G$-module structure on each $D^{q}(T, A)$ by putting $(x \cdot \alpha)\left(\tau_{1}, \ldots, \tau_{q}\right)=\alpha\left(\tau_{1} \cdot x, \ldots, \tau_{q} \cdot x\right) \cdot x^{-1}$. Identify $T$ with $F_{h}(G, C) \otimes_{c} \mathscr{L}(G)$. Then we see at once that $D^{q}(T, A)$ may be identified with $E^{q} \otimes_{i} F_{h}(G, A)$, where $E^{q}$ denotes the homogeneous component of degree $q$ of the exterior $C$-algebra built over the dual of $\mathscr{L}(G)$. The $G$-module structure of $D^{7}(T, A)$ is then given simply by $x \cdot(e \otimes f)=e \otimes\left(f \cdot x^{-1}\right)$. We topologize $D^{q}(T, A)$ by giving it the coarsest topology for which all the maps $\alpha \rightarrow \alpha\left(\tau_{1}, \ldots, \tau_{q}\right)$ are continuous from $D^{q}(T, A)$ to $F_{h}(G, A)$. Then $D^{q}(T, A)$, by the identification with $E^{q} \otimes{ }_{C} F_{h}(G, A)$, becomes topologically isomorphic with the direct sum of 
$n_{q}$ copies of $F_{h}(G, A)$, where $n_{q}$ is the dimension of $E^{q}$. Thus $D^{q}(T, A)$ is a complete locally convex topological vector space. We define an involution $f \rightarrow f^{\prime}$ on $F_{h}(G, A)$ by putting $f^{\prime}(x)=x \cdot f\left(x^{-1}\right)$. Evidently, this is a topological vector space automorphism of $F_{h}(G, A)$, and we have $f \cdot x^{-1}=\left(x \cdot f^{\prime}\right)^{\prime}$. Thus $F_{h}(G, A)$, with the $G$-module structure $f \rightarrow f \cdot x^{-1}$, is isomorphic with the $G$-module $F_{h}(G, A)$ as used before. Hence each $D^{q}(T, A)$ is a holomorphically injective holomorphic $G$-module. One verifies directly that $\delta$ is a continuous $G$-module homomorphism $D^{q}(T, A) \rightarrow D^{q+1}(T, A)$.

Now suppose that $G$ is a simply connected and solvable complex analytic group. Then, as a complex analytic manifold, $G$ is isomorphic with a $C^{n}$. Since the ordinary integration of continuous complex-valued functions on the closed interval $[0,1]$ extends to a continuous integral $F\left([0,1], D^{q}(G, A)\right.$ ) $\rightarrow D^{q}(G, A)$, it is clear that the usual constructive proof of the Poincare Lemma applies here and gives a continuous $C$-linear contracting homotopy $D^{q+1}(G, A) \rightarrow D^{q}(G, A), D^{\prime}(G, A) \rightarrow A$ for the complex $D(G, A)$, augmented by the map $A \rightarrow D^{o}(G, A)=F_{h}(G, A)$ sending each element $a$ of $A$ onto the constant function with value $a$. Hence the augmented complex $A \rightarrow D^{\circ}(G, A)$ $\rightarrow \cdots$ is a holomorphically injective resolution of the holomorphic $G$-module $A$, so that $H_{h}(G, A)$ may be identified with $H\left(D(T, A)^{G}\right)$, in this case. But $D^{q}(T, A)^{G}$ is simply $E^{a} \otimes c A^{*}$, where $A^{*}$ is the image of $A$ in $F_{h}(G, A)$ under the map $a \rightarrow f_{a}$, where $f_{a}(x)=x * a$. The $\mathscr{L}(G)$-module structure of $F_{h}(G, A)$ that is involved in the above formula for the differential operator $\delta$ stabilizes $A^{*}$ and corresponds, via the isomorphism $A \rightarrow A^{*}$, to the $\mathscr{L}(G)$-module structure of $A$ that is induced from the holomorphic $G$-module structure of $A$. Hence it is clear that the identification of $A^{*}$ with $A$ identifies the complex $D(T, A)^{G}$ with the standard complex whose homology is the Lie algebra cohomology space $H(\mathscr{L}(G), A)$. If $A$ is finite-dimensional this shows also that $H_{h}(G, A)$, with its natural structure as a topological vector space, is a finitedimensional Hausdorff space.

Generally, if $G$ is any complex analytic group and $A$ is a holomorphic $G$ module then we may regard a holomorphically injective resolution of $A$ as an acyclic complex $X$ of modules for the universal enveloping algebra $U(\mathscr{L}(G)$ ) of $\mathscr{L}(G)$. Given a $U(\mathscr{L}(G))$-injective resolution $Y$ of the $\mathscr{L}(G)$-module $A$, there is a unique homotopy class of homomorphisms of $U(\mathscr{L}(G))$-module 
complexes $X \rightarrow Y$, inducing the natural homomorphism $H_{h}(G, A) \rightarrow H(\mathscr{L}(G), A)$. Because of its naturality, this is compatible with the action of analytic group automorphisms on $H_{h}(G, A)$ and $H(\mathscr{L}(G), A)$. Combining our last result for simply connected solvable groups with Theorem 3.1 , we have therefore the following theorem.

THEOREM 4.1. Suppose that the complex analytic group $G$ is a semidirect product $P \cdot K$, where $K$ is a simply connected solvable analytic group, normal in $G$, and $P$ has an ample connected compact subgroup $Q$. Let $A$ be a finite-dimensional holomorphic G-module. Then the restriction map $H_{h}(G, A) \rightarrow\left(H_{h}(K, A)\right)^{\theta}$, followed by the natural map $\left(H_{\imath}(K, A)\right)^{\Theta} \rightarrow(H(\mathscr{L}(K), A))^{G}$ is an isomorphism of $H_{h}(G, A)$ onto $(H(\mathscr{L}(K), A))^{G}$.

5. Linear complex analytic groups. First, let us consider an irreducible algebraic complex linear group $G$. Let $N(G)$ denote the maximum normal unipotent subgroup of $G$. There is a fully reducible algebraic subgroup $P$ of $G$ such that $G$ is the semidirect product $P \cdot N(G)$. Moreover, $P$ has a connected compact subgroup $Q$ such that the real Lie algebra of $Q$ spans the Lie algebra of $P$ over $C$ [5, Th. 6.1]. Let $A$ be a finite-dimensional rational $G$-module. Regarding $G$ as a complex analytic group and $A$ as a holomorphic $G$-module, and noting that $N(G)$ is a simply connected solvable (even nilpotent) complex analytic group, we may apply Theorem 4.1 to conclude that the natural map $H_{h}(G, A) \rightarrow(H(\mathscr{L}(N(G)), A))^{G}$ is an isomorphism.

On the other hand, let us consider the rational cohomology of $G$, as treated in [1]. This is based on the category of rational $G$-modules, i.e., sums of finite-dimensional representation spaces of rational representations of $G$, and $C$-linear $G$-module homomorphisms, and it does not involve any topology on the rational $G$-modules. If $H_{\rho}$ denotes rational cohomology and $A$ is a finitedimensional rational $G$-module one has, analogously to Theorem 4.1 , that the natural map $H_{\rho}(G, A) \rightarrow(H(\mathscr{L}(N(G)), A))^{\theta}$ is an isomorphism [1, Th. 5.2].

Now, given any rational $G$-module $M$, we may endow $M$ with the coarsest topology making all $C$-linear maps $M \rightarrow C$ continuous. Then $M$ evidently becomes a topological vector space and, since it is a locally finite $G$-module, it thus becomes a holomorphic $G$-module. Moreover, every exact sequence of such modules is evidently strongly exact. It follows that there is a natural 
map $H_{\rho}(G, M) \rightarrow H_{h}(G, M)$, obtained in the usual way from any continuous homomorphism of complexes from a rationally injective resolution of $M$ into a holomorphically injective resolution of $M$. Hence our above results for $H_{h}(G, A)$ and for $H_{\rho}(G, A)$ give the following result.

Theorem 5.1. Let $G$ be an irreducible algebraic complex linear group, and let $A$ be a finite-dimensional rational G-module. Then the natural map $H_{\rho}(G, A) \rightarrow H_{h}(G, A)$ is an isomorphism.

Now let $G$ be any linear complex analytic group. We recall that $G$ has a semidirect product decomposition $G=P \cdot K$, where $K$ is a simply connected solvable normal complex analytic subgroup of $G$ and $P$ is a reductive complex analytic group, i.e., a group of the same type as the group denoted by $P$ in our above discussion of algebraic linear groups [2, Th. 4.2]. By an algebraic hull of $G$ we mean an algebraic complex linear group $L$ containing $G$ as a complex analytic subgroup and such that $G$ is algebraically dense in $L$. Let $A$ be a finite-dimensional holomorphic $G$-module. It is known from the elementary theory of the algebra of representative functions on $G$ that there is an algebraic hull $G^{*}$ of $G$ having the following two properties: (1) every complex analytic homomorphism of $G$ into the additive group $C$ of the complex numbers extends to a rational homomorphism of $G^{*}$ into $C$; (2) the given representation of $G$ on $A$ extends to a rational representation of $G^{*}$ on $A$. Moreover, every algebraic hull $G^{*}$ of $G=P \cdot K$ satisfying (1) has a semidirect product decomposition $G^{*}=P^{*} \cdot K$, in the sense of complex analytic groups, where $P^{*}$ is a fully reducible algebraic subgroup of $G^{*}$ containing $P$. For this, see the proof of Th. 4.5 and the note following it in [4].

By Theorem 4.1, the natural map $H_{h}\left(G^{*}, A\right) \rightarrow(H(\mathscr{L}(K), A))^{G^{*}}$ is an isomorphism. Using that the adjoint representation of $G^{*}$ is a rational representation and that the representation of $G^{*}$ on $A$ is a rational representation, one sees easily by examining the standard complex giving $H(\mathscr{L}(K), A)$ that $H(\mathscr{L}(K), A)$ is a rational $G^{*}$-moduie. Since $G$ is algebraically dense in $G^{*}$, it follows therefore that $(H(\mathscr{L}(K), A))^{\sigma^{*}}=(H(\mathscr{L}(K), A))^{G}$.

Now we have a natural map $H_{\rho}\left(G^{*}, A\right) \rightarrow H_{h}(G, A)$, namely the natural map $H_{\rho}\left(G^{*}, A\right) \rightarrow H_{h}\left(G^{*}, A\right)$ followed by the restriction map $H_{h}\left(G^{*}, A\right) \rightarrow$ $H_{h}(G, A)$, and we know from Theorem 5.1 that the first of these two maps is an isomorphism. If we follow up the second map with the natural map $H_{h}(G, A)$ 
$\rightarrow(H(\mathscr{L}(K), A))^{G}=(H(\mathscr{L}(K), A))^{G *}$ we obtain the natural map $H_{h}\left(G^{*}, A\right)$ $\rightarrow(H(\mathscr{L}(K), A))^{G *}$. Since these maps are isomorphisms, we have therefore the following result.

Theorem 5.2. Let $G$ be a linear complex analytic group, $A$ a finite-dimensional holomorphic G-module. There is an algebraic hull $G^{*}$ of $G$ such that the representation of $G$ on $A$ extends to a rational representation of $G^{*}$ on $A$ and every complex analytic homomorphism of $G$ into $C$ extends to a rational homomorphism of $G^{*}$ into $C$. For such an algebraic hull $G^{*}$, the natural map $H_{\rho}\left(G^{*}, A\right) \rightarrow H_{h}(G, A)$ is an isomorphism.

The following example shows that, in Theorem 5.2, the condition that the complex analytic homorphisms of $G$ into $C$ extend to rational homomorphisms of $G^{*}$ into $C$ is not superfluous. Let $G$ be the additive group $C$ of the complex numbers. If we identify $C$ with the multiplicative group of matrices $\left(\begin{array}{ll}e^{c} & 0 \\ 0 & e^{i c}\end{array}\right)$ we see that $G$ has an algebraic hull $G^{*}$ that is isomorphic with the direct product of two copies of the multiplicative group of the non-zero complex numbers. Thus $G^{*}$ is a fully reducible algebraic linear group, whence $H_{\mathrm{p}}^{n}\left(G^{*}, A\right)=(0)$ for all rational $G^{*}$-modules $A$ and all $n>0$. On the other hand, by Theorem $4.1, H_{h}(G, A)$ is isomorphic with $H(\mathscr{L}(G), A)$ for every finitedimensional holomorphic $G$-module $A$. In particular, if $A$ is the 1-dimensional trivial $G^{*}$-module we have therefore $H_{h}^{1}(G, A) \neq(0)$.

\section{REFERENCES}

[0] N. Bourbaki, Integration, Chap. III, Hermann, Paris, 1952.

[1] G. Hochschild, Cohomology of algebraic linear groups, Illinois Jour. of Math., vol. 5 (1961), pp. 492-519.

[2] G. Hochschild and G. D. Mostow, Representations and representative functions of Lie groups, III, Annals of Math., vol. 70 (1959), pp. 85-100.

[3] G. Hochschild and G. D. Mostow, Cohomology of Lie groups, Illinois Jour. of Math., vol. 6 (1962), pp. 367-401.

[4] G. Hochschild and G. D. Mostow, Affine embeddings of complex analytic homogeneous spaces, Amer. Jour. of Math., vol. 87 (1965), pp. 807-839.

[5] G. D. Mostow, Self-adjoint groups, Annals of Math., vol. 62 (1955), pp. 44-55.

University of California, Berkeley, and Yale University 